Effect of difference i $n$ i nt er act i on strengt $h$ on two-di mensi onal I at i ce structure in a bi nary system wi th DNA nanoparti cles

\begin{tabular}{|l|l|}
\hline 著者 & Tanaka Kei ta, Kat suno H r oyasu, Sat o Masahi de \\
\hline $\begin{array}{l}\text { j our nal or } \\
\text { publ i cat i on ti tl e }\end{array}$ & Japanese Jour nal of Appl i ed Physi cs \\
\hline vol une & 56 \\
\hline page range & 075001 \\
\hline year & $2017-06-21$ \\
\hline URL & ht t p: //doi . or g/10. 24517/00028596 \\
\hline
\end{tabular}




\title{
Effect of difference in interaction strength on two-dimensional lattice structure in a binary system with DNA nanoparticles
}

\author{
Keita Tanaka ${ }^{1}$, Hiroyasu Katsuno ${ }^{2}$, and Masahide Sato ${ }^{3}$ \\ ${ }^{1}$ Graduate School of Natural Science and Technology, Kanazawa University, Kanazawa 920-1192, Japan \\ ${ }^{2}$ Department of Physical Sciences, Ritsumeikan University, Kusatsu, Shiga 525-8577, Japan \\ ${ }^{3}$ Information Media Center, Kanazawa University, Kanazawa 920-1192, Japan
}

\begin{abstract}
Keeping two-dimensional lattice structures formed by nanoparticles covered with DNA in mind, we carry out Brownian dynamics simulations to study the effect of interaction strength on a two-dimensional lattice structure formed in a binary system. In our previous study [H. Katsuno, Y. Maegawa, and M. Sato, J. Phys. Soc. Jpn. 85, 074605 (2016)], we carried out simulations using the Lennard-Jones potential, in which the difference in interaction length was taken into account. When the interaction length between different species, $\sigma^{\prime}$, is smaller than that between the same species, $\sigma$, various lattice structures were formed with changing the ratio $\sigma^{\prime} / \sigma$. In this paper, taking the difference in the interaction strength into account, we study the effect of the difference in interaction strength on the two-dimensional lattice structure.
\end{abstract}

\section{Introduction}

A nanoparticle covered with DNA strands, which we call the DNA nanoparticle, is one of promising building blocks for fabricating nanostructures. Since the DNA strands attaching to DNA nanoparticles are designed freely, we can control the interaction between the particles independently of particle size and particle shape according to the purpose.

There are many experiments on the formation of three-dimensional structures. ${ }^{1-5}$ When DNA nanoparticles are connected to each other by self-complementary linkers, the fcc structure is formed. When two kinds of non-self-complementary linkers are used to connect the DNA particles, the system is regarded as a binary system and the bcc structure is formed. The $\mathrm{NaCl}$ lattice structure is formed when the particles with both self-complementary and non-self-complementary linkers are used. ${ }^{4} \mathrm{CsCl}, \mathrm{AlB}_{2}, \mathrm{Cr}_{3} \mathrm{Si}$, and $\mathrm{Cs}_{6} \mathrm{C}_{60}$ lattices can also be formed by controlling particle size. ${ }^{5}$

Recently, Isogai and coworkers have studied the formation of a two-dimensional lattice structure in a binary system. ${ }^{6,7}$ They showed that the lattice structure can be changed from the triangular lattice to the square one by controlling the magnesium 
ion density in a solution. ${ }^{7}$ They proposed that DNA strands are bundled with a high density of magnesium ions, which changes the coordination number and the particles covered with the DNA strands form a square lattice. However, it has not been clarified whether their proposal is correct.

Previously, we studied the formation of two-dimensional lattice structures in a binary system, ${ }^{8}$ keeping the two-dimensional lattice structure formed by DNA nanoparticles in mind. For simplicity, we used the Lennard-Jones (LJ) potential as the interaction potential. We assumed that the interaction length between different species, $\sigma^{\prime}$, is smaller than that between the same species, $\sigma$. In Ref. 8, we showed the possibility of changing the lattice structure by controlling $\sigma^{\prime} / \sigma$ : a stable lattice structure is successively changed from a triangular lattice to a square lattice, a honeycomb lattice and, a rectangular lattice with decreasing ratio $\sigma^{\prime} / \sigma$.

In our previous study, ${ }^{8}$ we neglected the difference in interaction strength: the interaction strength between the same species, $\epsilon$, is equal to that between different species, $\epsilon^{\prime}$. However, the difference in interaction strength probably affects the stable lattice structure. Thus, in this paper, we take into account the difference between $\epsilon^{\prime}$ and $\epsilon$, and study the two-dimensional lattice formed in the binary system. In Sect. 2, we introduce our model. In Sect. 3, we show the results of Brownian dynamics simulations. We show how the lattice structure is changed by $\epsilon^{\prime} / \epsilon$. In Sect. 4 , we summarize our results.

\section{Model}

In experiments, ${ }^{6,7}$ DNA nanoparticles are adsorbed on a cationic supported lipid bilayer by electrostatic coupling between DNA strands and cationic lipids. Two-dimensional diffusion of the DNA nanoparticles on the lipids occurs since the lipids move similarly to a fluid. Thus, the motion of the $i$ th DNA nanoparticle is given by

$$
m \frac{d^{2} \boldsymbol{r}_{i}}{d t^{2}}=-\xi \frac{d \boldsymbol{r}_{i}}{d t}+\boldsymbol{F}_{i}+\boldsymbol{F}_{i}^{\mathrm{B}}
$$

where $m$ is the mass of the DNA nanoparticle, $\boldsymbol{r}_{i}$ is the position of the $i$ th particle, and $\xi$ is the frictional coefficient. $\boldsymbol{F}_{i}^{\mathrm{B}}$ and $\boldsymbol{F}_{i}$ represent the thermal noise and the sum of inertial forces, respectively. $\boldsymbol{F}_{i}^{\mathrm{B}}$ satisfies $\left\langle\boldsymbol{F}_{i}^{\mathrm{B}}\right\rangle=\mathbf{0}$ and $\left\langle F_{i, k}^{\mathrm{B}}(t) F_{j, l}^{\mathrm{B}}\left(t^{\prime}\right)\right\rangle=2 \xi k_{\mathrm{B}} T \delta_{i j} \delta_{k l} \delta\left(t-t^{\prime}\right)$, where $F_{i, k}^{\mathrm{B}}(t)$ represents the $k$-direction component of thermal noise at time $t . \boldsymbol{F}_{i}$ is given by the gradient of the interaction potential $U\left(r_{i j}\right)$ as

$$
\boldsymbol{F}_{i}=\sum_{i \neq j} \nabla U\left(r_{i j}\right)
$$


where $r_{i j}=\left|\boldsymbol{r}_{i}-\boldsymbol{r}_{j}\right|$. It is suggested that the DNA nanoparticle can be treated as a fuzzy sphere in Ref. 4, but an appropriate potential for expressing the effect of the attraction by DNA strands is not obvious. At least, taking into account the attraction by DNA strands and the repulsion by the excluded volume effect of nanoparticles is necessary. Thus, as a simple potential, we use the Lennard--Jones (LJ) potential as the interaction potential:

$$
U\left(r_{i j}\right)=4 \epsilon_{i j}\left[\left(\frac{\sigma_{i j}}{r_{i j}}\right)^{12}-\left(\frac{\sigma_{i j}}{r_{i j}}\right)^{6}\right],
$$

where $\epsilon_{i j}$ and $\sigma_{i j}$ represent the interaction strength and interaction length, respectively. Nanoparticles are exposed to a sharp short-range repulsion at $r_{i j}<2^{1 / 6} \sigma_{i j}$ and a gradual long-range attraction at $r_{i j}>2^{1 / 6} \sigma_{i j}$. When $r_{i j} \geq 3 \sigma_{i j}$, the attractive force is negligibly small. Thus, the LJ potential is suitable for hard spheres covered with flexible DNA strands as a first approximation.

We consider a binary system, in which the numbers of two species are the same. In our previous study, ${ }^{8}$ we assumed that the interaction length for different species, $\sigma^{\prime}$, was shorter than that for the same species, $\sigma$. The interaction strength $\epsilon_{i j}$ was independent of the combination of species. In this paper, we assume that both $\epsilon_{i j}$ and $\sigma_{i j}$ depend on the combination of species: $\sigma_{i j}=\sigma$ and $\epsilon_{i j}=\epsilon$ for the combination of the same species and $\sigma_{i j}=\sigma^{\prime}<\sigma$ and $\epsilon_{i j}=\epsilon^{\prime}>\epsilon$ for the combination of different species. Experimentally, ${ }^{1-5} \epsilon$ is negligibly small in a binary system, so that decreasing $\epsilon$ might be more practical than increasing $\epsilon^{\prime}$ to realize $\epsilon / \epsilon^{\prime} \rightarrow 0$. In our simulation, however, decreasing temperature is necessary to prevent thermal disordering when we decrease $\epsilon$. Thus, we increase $\epsilon^{\prime}$ to avoid the complication of temperature tuning to realize $\epsilon / \epsilon^{\prime} \rightarrow 0$.

Since DNA particles are adsorbed on the lipid bilayer in experiments, ${ }^{6,7}$ the friction is probably large. Thus, we neglect $m d^{2} \boldsymbol{r} / d t^{2}$, and solve Eq. (1) numerically using a simple difference equation given by ${ }^{9}$

$$
\tilde{r}_{i}\left(\tilde{t}_{n+1}\right)=\tilde{r}_{i}\left(\tilde{t}_{n}\right)+\tilde{\boldsymbol{F}}_{i} \Delta \tilde{t}+\Delta \tilde{\boldsymbol{r}}_{i}^{\mathrm{B}},
$$

where $\tilde{\boldsymbol{r}}_{i}=\boldsymbol{r}_{i} / \sigma, \tilde{t}_{n}=\epsilon t_{n} /\left(\xi \sigma^{2}\right), \tilde{\boldsymbol{F}}_{i}=\sigma \boldsymbol{F}_{i} / \epsilon$, and $\tilde{t}_{n+1}=\tilde{t}_{n}+\Delta \tilde{t}$. The displacement caused by a random force, $\Delta \tilde{\boldsymbol{r}}_{i}^{\mathrm{B}}$, satisfies $\left\langle\Delta \tilde{\boldsymbol{r}}_{i}^{\mathrm{B}}\right\rangle=\mathbf{0}$ and $\left\langle\Delta \tilde{r}_{i, k}^{\mathrm{B}}\left(t_{n}\right) \Delta \tilde{r}_{j, l}^{\mathrm{B}}\left(t_{m}\right)\right\rangle=$ $2 k_{\mathrm{B}} T \delta_{i j} \delta_{k l} \delta_{m n} / \epsilon$, where $\Delta \tilde{r}_{i, k}^{\mathrm{B}}\left(t_{n}\right)$ represents the component of $\Delta \tilde{\boldsymbol{r}}_{i}^{\mathrm{B}}$ in the $k$-th direction. We carry out the simulations in a square system with the system size $L \times L$ under the periodic boundary condition.

In this paper, we fix the value of $\sigma^{\prime} / \sigma$ and investigate how a lattice structure is 
changed by $\epsilon^{\prime} / \epsilon$. To specify the type of lattice structures, we carry out simulations using some individual initial conditions, and calculate the radial distribution function $g(r)$ and the parameter of local rotational symmetry $\phi_{k}$ by averaging them over individual samples. The radial distribution function $g(r)$ is defined as

$$
g(r)=\frac{1}{N_{\mathrm{s}}} \frac{1}{N} \sum_{m}^{N_{\mathrm{s}}} \sum_{i}^{N} \frac{n_{i, m}}{2 \pi r \delta r},
$$

where $n_{i, m}$ represents the number of particles between the distance $r$ and $r+\delta r$ for the $i$ th particle in the $m$ th sample. $N$ is the number of particles in a sample and $N_{\mathrm{s}}$ represent the number of individual samples. In our simulation, $N, N_{\mathrm{s}}$, and $\delta r$ are set to be 512,10 , and $10^{-2}$, respectively.

The parameter $\phi_{k}$, which represents the averaged $k$-hold rotational symmetry, is defined as

$$
\phi_{k}=\frac{1}{N_{\mathrm{s}}} \frac{1}{N} \sum_{m}^{N_{\mathrm{s}}} \sum_{i}^{N} \phi_{k}^{m}(i),
$$

where $\phi_{k}^{m}(i)$ is the local $k$-hold rotational symmetry around the $i$ th particle in the $m$ th sample. $\phi_{k}^{m}(i)$ is given by ${ }^{10-14}$

$$
\phi_{k}^{m}(i)=\frac{1}{N_{\mathrm{n}}^{m}(i)}\left|\sum_{j=1}^{N_{\mathrm{n}}^{m}(i)} \mathrm{e}^{\mathrm{i} k \theta_{i j}^{m}}\right|,
$$

where $N_{\mathrm{n}}^{m}(i)$ and $\theta_{i j}^{m}$ are the number of neighbors around the $i$ th particle and the angle representing the direction of the $j$ th particle in the $m$ th sample, respectively.

In all our simulations, temperature, system size, and interaction length ratio $\sigma^{\prime} / \sigma$ are set to be $k_{\mathrm{B}} T / \epsilon=0.1$, and $L=23.97$, and $\sigma^{\prime} / \sigma=0.45$, respectively. We carry out simulations using various values of $\epsilon^{\prime} / \epsilon$ and study how the structure is changed by the interaction strength ratio.

\section{Results and discussion}

In our previous study, ${ }^{8}$ the lattice structure is changed from the triangular lattice to the square lattice, honeycomb lattice, and the rectangular lattice with decreasing $\sigma^{\prime} / \sigma$. When $\sigma^{\prime} / \sigma$ is too small, the potential we use in our simulations is not realistic because it is necessary to consider the effect of steric hindrance. However, our purpose is to investigate how $\epsilon^{\prime} / \epsilon$ affects lattice structures. Thus, we set $\sigma^{\prime} / \sigma$ to be 0.45 , with which the rectangular lattice is formed when $\epsilon^{\prime} / \epsilon=1$, and carry out simulations at a low temperature. 


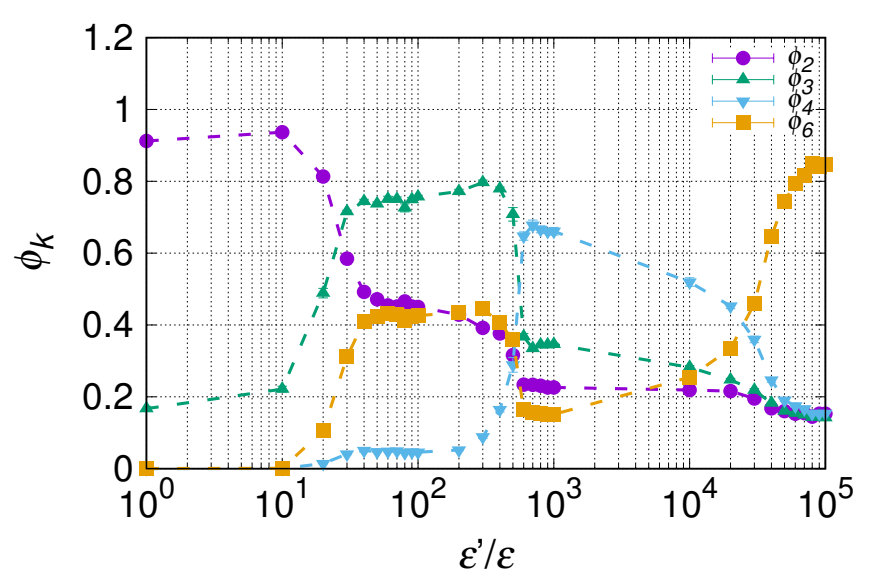

Fig. 1. (color online) Dependence of $\phi_{k}$ with $k=2,3,4$, and 6 on $\epsilon^{\prime} / \epsilon$.

To study how the lattice structure is changed by $\epsilon^{\prime} / \epsilon$, first we investigate the change in rotational symmetry caused by $\epsilon^{\prime} / \epsilon$. Figure 1 shows the dependence of $\phi_{k}$ with $k=2$, 3 , 4 , and 6 on $\epsilon^{\prime} / \epsilon . \phi_{2}$ is the largest parameter when $\epsilon^{\prime} / \epsilon=1$, which shows the formation of the rectangular lattice. ${ }^{8}$ When $\epsilon^{\prime} / \epsilon>10, \phi_{2}$ starts decreasing, and $\phi_{3}$ starts increasing instead. $\phi_{3}$ becomes the largest parameter when $30<\epsilon^{\prime} / \epsilon<5 \times 10^{2}$, which shows the formation of the honeycomb lattice. Then, $\phi_{3}$ decreases and $\phi_{4}$ increases sharply with increasing $\epsilon^{\prime} / \epsilon$. The largest parameter is $\phi_{4}$ when $6 \times 10^{2}<\epsilon^{\prime} / \epsilon<2 \times 10^{4}$ and $\phi_{6}$ when $3 \times 10^{4}<\epsilon^{\prime} / \epsilon$. These results show the formation of the square lattice and the triangular lattice, respectively. The range of the changes in $\epsilon^{\prime} / \epsilon$ is wide. In Eq. (4), the amplitude of displacement caused by the random force is proportional to $k_{\mathrm{B}} T / \epsilon$. In our simulations, $k_{\mathrm{B}} T / \epsilon$ is kept constant. Experimentally, a similar condition is probably realized by decreasing $\epsilon$ with increasing $\epsilon^{\prime}$ and tuning temperature. Decreasing interaction strength is possible by changing the DNA strands. Thus, we think that our range of parameter of interaction energies is probably possible in an experiment.

The changes in the rotational symmetries show that the rectangular lattice successively changes to the honeycomb lattice, square lattice, and triangular lattice with increasing $\epsilon^{\prime} / \epsilon$. Hereafter, we show snapshots and the radial distribution functions for some typical $\epsilon^{\prime} / \epsilon$ values to confirm the changes in the lattice structure.

Figure 2 shows a snapshot of the rectangular lattice, in which the density of particles is given by $\pi N(\sigma / 2)^{2} / L^{2}=0.7$ if all the particles are isolated. In our previous study, ${ }^{8}$ two types of particles individually form the triangular lattices with $\sigma^{\prime} / \sigma=0.45$, where a different type of particle is present in the middle point on a side of the triangular lattice. In the rectangular lattice, the ratio of the long side formed by the pair of different 


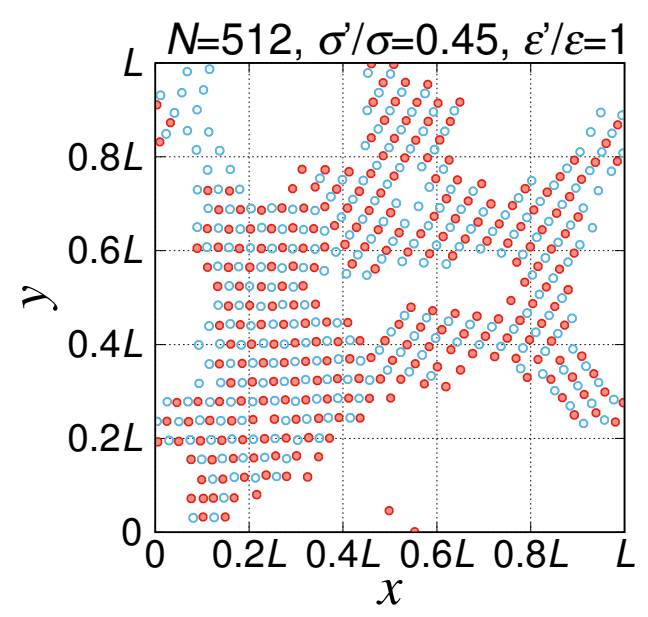

Fig. 2. (color online) Snapshot of a rectangular lattice. Parameters are given by $\sigma^{\prime} / \sigma=0.45$, $\epsilon^{\prime} / \epsilon=1$, and $k_{\mathrm{B}} T / \epsilon=0.1$. The number of particles $N$ is 512 and the length of one side of the square system satisfies $L / \sigma=23.97$. We use open circles and solid circles to distinguish two species, where the sizes of the circles are smaller than 0.5 to show the positions of the species clearly.

species to the short side formed by the same species is $\sqrt{3}$. Thus, the lengths of the short and long lattice constants are about $0.45 r^{*}$ and $0.45 \sqrt{3} r^{*}$ with $r^{*}=2^{1 / 6} \sigma$, respectively. The actual area fraction is much lower than 0.7 as shown in Fig. 2.

To confirm the formation of the rectangular lattice, we calculate the radial distribution function $g(r)$. Figure 3(a) shows the radial distribution function, for which we use the same parameters as those in Fig. 2. In Fig. 3(b), we show the contributions of the same and different species to $g(r)$, respectively. To see the higher-order peak clearly, we show $2 \pi r \delta r g(r)$ in the vertical axis. The peaks appear at $r=0.486 r^{*}, r=0.878 r^{*}$, and $r=0.985 r^{*}$. The first and second peaks are formed by different species, and the third peak is formed by the same species [Fig. 3(b)]. From the radial distribution function, the formation of the rectangular lattice ${ }^{8}$ is confirmed .

Since temperature is sufficiently low, the rectangular lattice is formed in Fig. 2. If we increase the interaction strength keeping the temperature low, disordering caused by thermal noise is prevented. Thus, by setting $k_{\mathrm{B}} T / \epsilon$ to 0.1 and increasing $\epsilon^{\prime} / \epsilon$, we carry out simulations. Figure 4 shows the snapshots with some $\epsilon^{\prime} / \epsilon^{\prime}$ s. With increasing $\epsilon^{\prime} / \epsilon$, the lattice structure is changed from the rectangular lattice to the honeycomb lattice at $\epsilon^{\prime} / \epsilon=10^{2}$ [Fig. 4(a)], the square lattice at $\epsilon^{\prime} / \epsilon=10^{3}$ [Fig. 4(b)], and the triangular lattice at $\epsilon^{\prime} / \epsilon=10^{5}$ [Fig. $4(\mathrm{c})$ ].

Figures 5-7 show $g(r)$ for those $\epsilon^{\prime} / \epsilon^{\prime}$ s. Figure 5 shows $g(r)$ with $\epsilon^{\prime} / \epsilon=10^{2}$. Since 

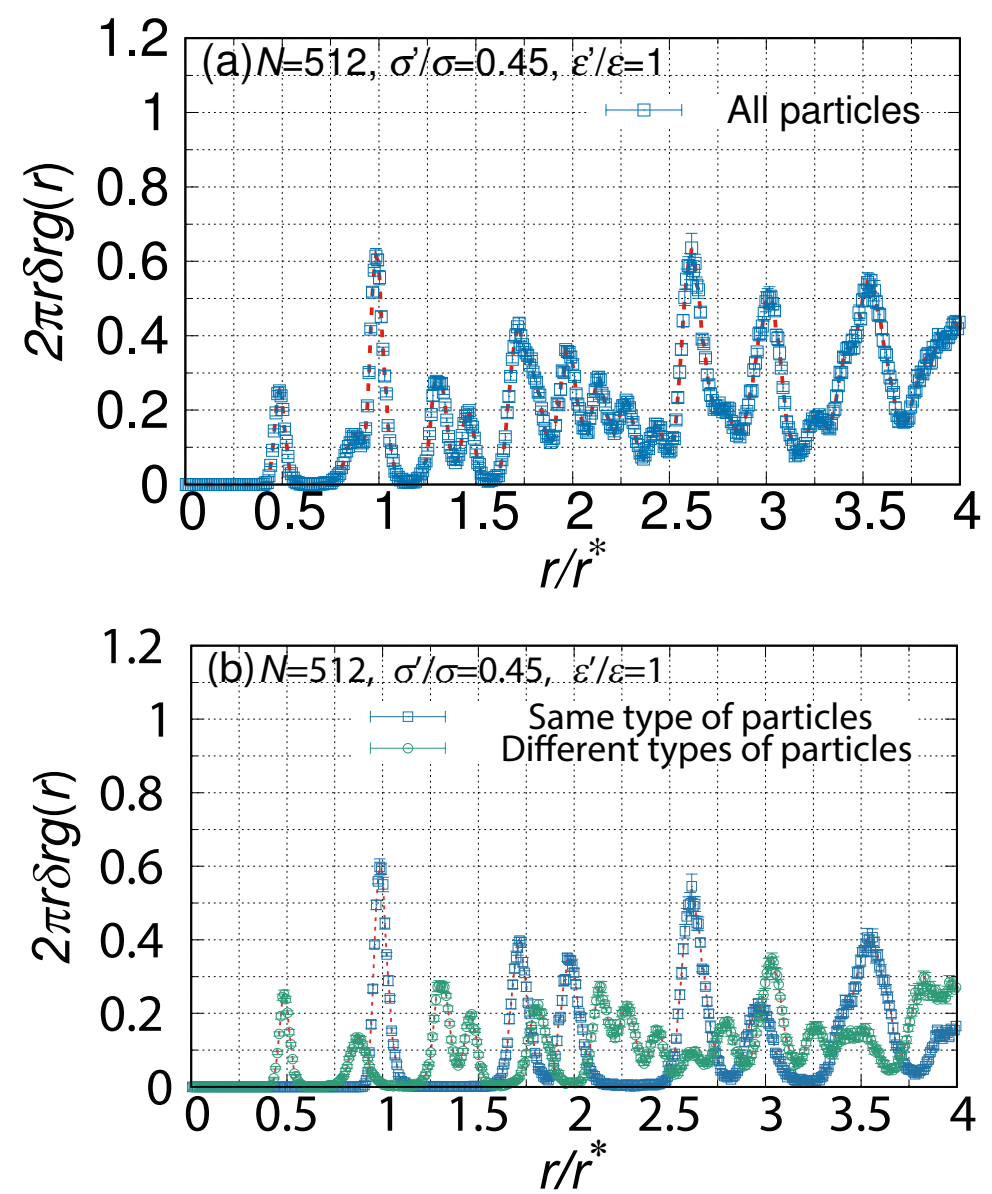

Fig. 3. (color online) (a) Radial distribution function $g(r)$ and (b) the contributions of the same and different species to $g(r)$, where parameters are given by $\sigma^{\prime} / \sigma=0.45, k_{\mathrm{B}} T / \epsilon=0.1$, and $\epsilon^{\prime} / \epsilon=1$. To see the higher-order peak clearly, we show $2 \pi r \delta r g(r)$ in the vertical axis. The data are the averages of 10 samples.

the interaction strength $\epsilon^{\prime}$ is much larger than that in Fig. 2, the effect of thermal noise decreases relatively. Thus, the peaks are sharper than those in Fig. 3. The positions of the first, second, and third peaks are $0.459 r^{*}, 0.807 r^{*}$, and $0.949 r^{*}$, respectively. The first and third peaks correspond to different species and the second peak corresponds to the same species [Fig. 5(b)]. We find the formation of the honeycomb lattice from these peaks.

Figure 6 shows $g(r)$ with $\epsilon^{\prime} / \epsilon=10^{3}$. The positions of the first, second, and third peaks are $0.459 r^{*}, 0.664 r^{*}$, and $0.922 r^{*}$, respectively. The first and third peaks consist of different species and the second peak consists of the same species [Fig. 6(b)]. The peaks positions are close to those of the square lattice with the lattice constant of $0.45 r_{0}$.

Figure 7 shows $g(r)$ with $\epsilon^{\prime} / \epsilon=10^{5}$. The positions of the first, second and third 

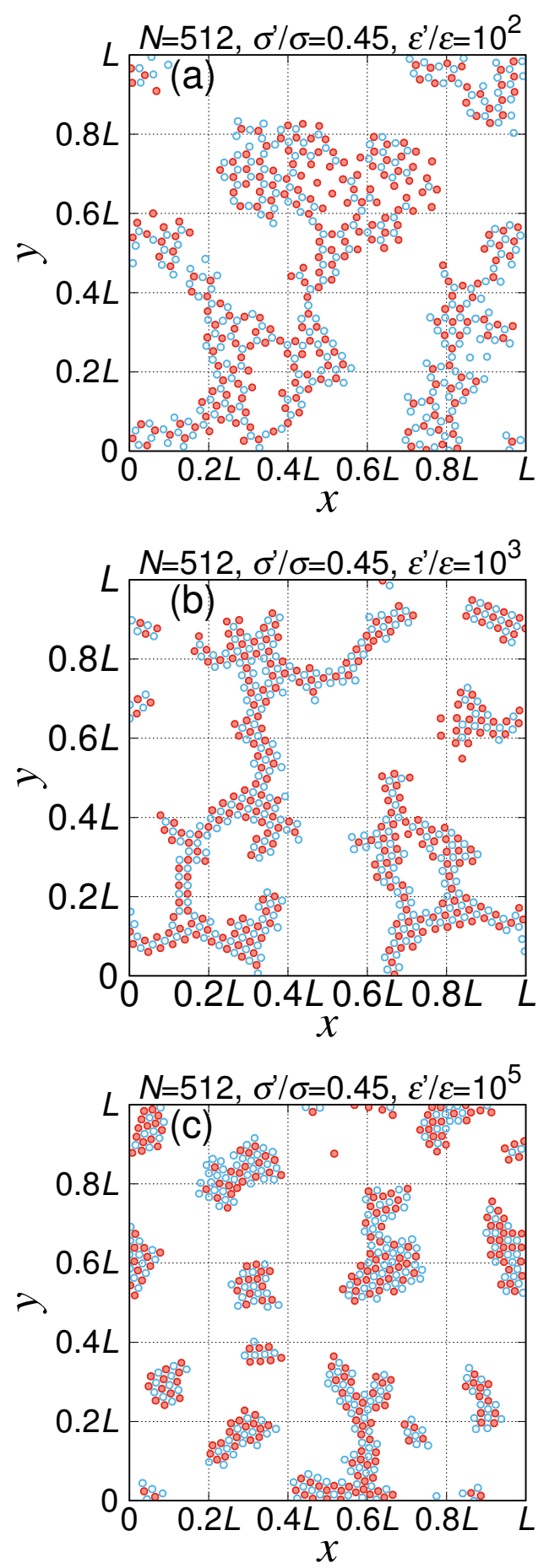

Fig. 4. (color online) Snapshots of a rectangular lattice. Parameters are given by $\sigma^{\prime} / \sigma=0.45$ and $k_{\mathrm{B}} T / \epsilon=0.1$. The values of the ratio $\epsilon^{\prime} / \epsilon$ are (a) $10^{2}$, (b) $10^{3}$, and (c) $10^{5}$. The number of particles $N$ is 512 and the length of one side of the square system satisfies $L / \sigma=23.97$. We use open circles and solid circles to distinguish two species, where the sizes of the circles are smaller than 0.5 to show the positions of the species clearly. 

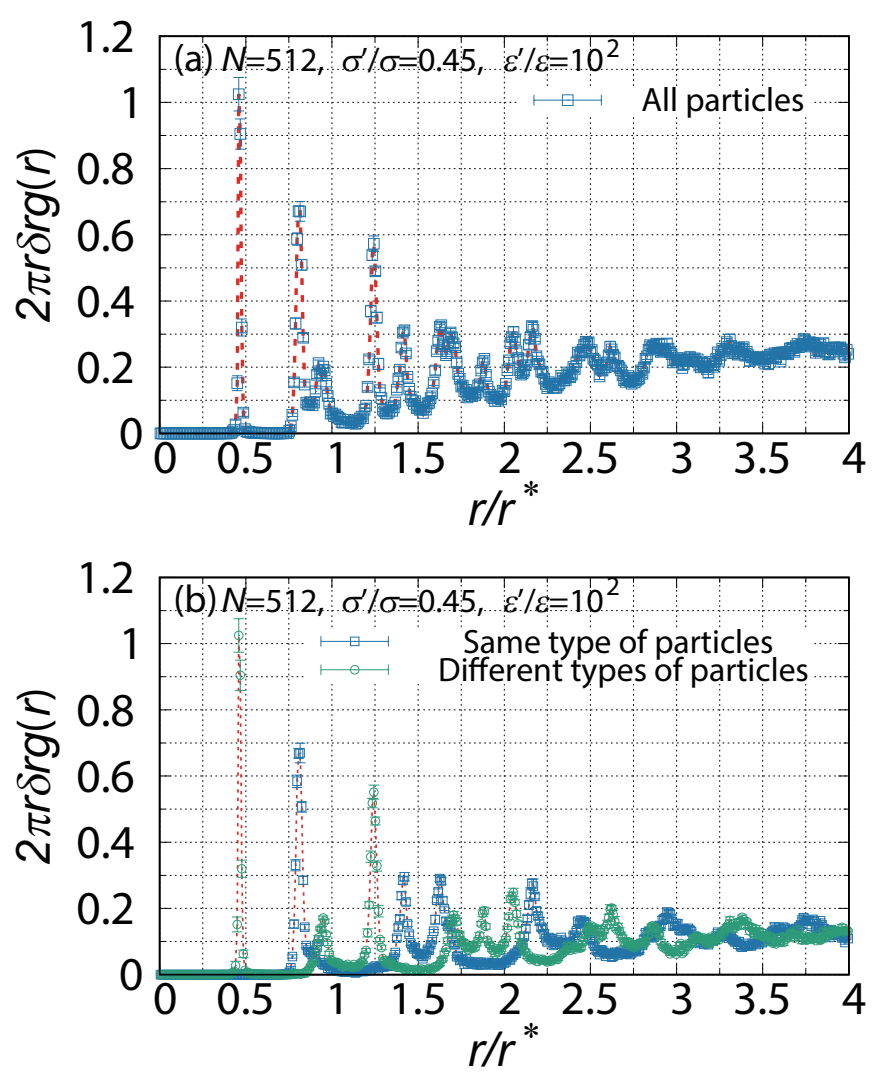

Fig. 5. (color online) (a) Radial distribution function $g(r)$ and (b) the contributions of the same and different species to $g(r)$, where $\sigma^{\prime} / \sigma=0.45, k_{\mathrm{B}} T / \epsilon=0.1$, and $\epsilon^{\prime} / \epsilon=10^{2}$. To see the higher-order peak clearly, we show $2 \pi r \delta r g(r)$ in the vertical axis. The data are the averages of 10 samples.

peaks are $0.450 r^{*}, 0.807 r^{*}$, and $0.905 r^{*}$, respectively. The peaks correspond to both the same and different species. From the peaks in Fig 7(b) the ratios of the contribution of different species to that of the same species in the first, second, and third peaks are given by 6.0 to $1,0.35$ to 1 , and 0.84 to 1 , respectively. The positions of the peaks are close to those in the triangular lattice with the lattice constant $0.45 r^{*}$.

In our previous study, ${ }^{8}$ the change in the lattice structure induced by $\sigma^{\prime} / \sigma$ is understood by the rough estimation of interaction energies. Here, we estimate the interaction energies similarly to explain the change in lattice structure induced by the change in $\epsilon^{\prime} / \epsilon$. In our simulations, the nearest neighbors consist of different species except for the triangular lattice. Since $\epsilon^{\prime} \geq \epsilon$, we set the distance to the nearest neighboring particles $r_{0}$ to be $2^{1 / 6} \sigma^{\prime}$. Figure $8(\mathrm{a})$ shows the structure of the rectangular lattice expected from Fig. 2. We sum up the interaction energies in the minimum unit surrounded by the red dotted lines. The distance between the nearest neighbors, e. g., the particles b and c, is $r_{1}=r_{0}$. The distance between the second nearest neighbors, e. g., the particles a 

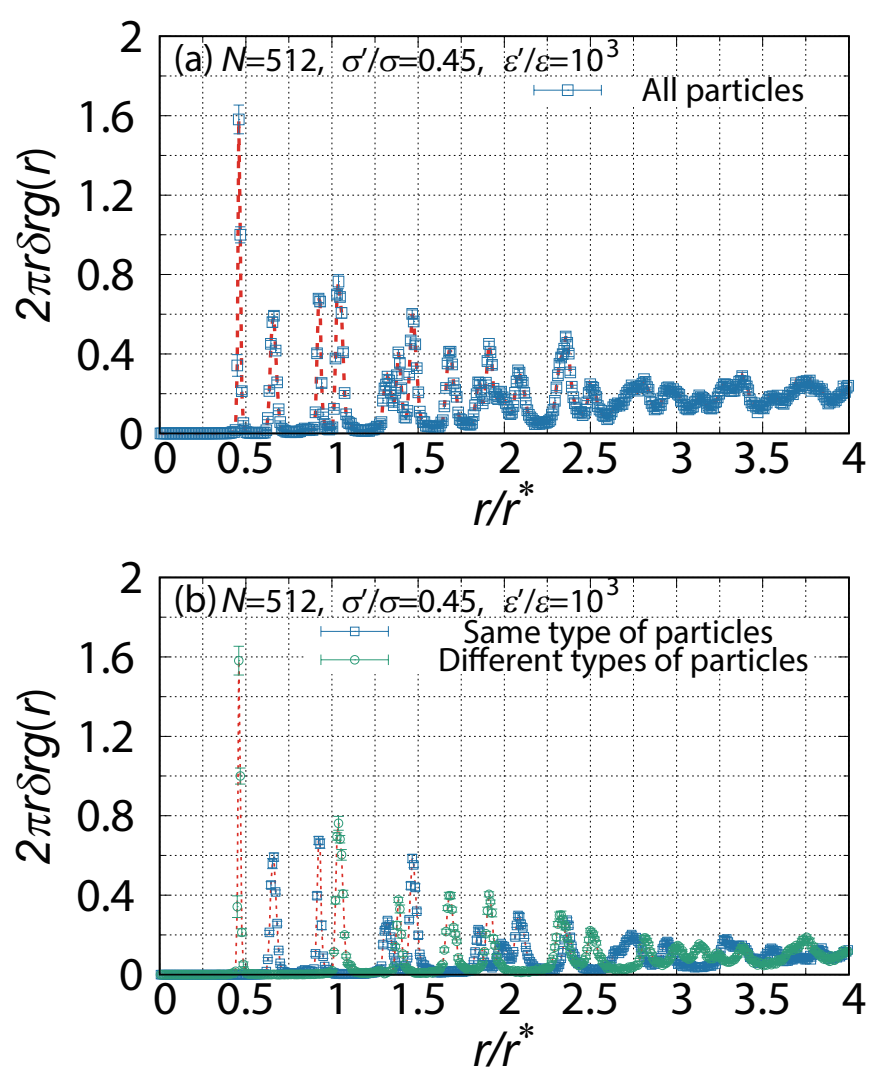

Fig. 6. (color online) (a) Radial distribution function $g(r)$ and (b) the contributions of the same and different species to $g(r)$, where $\sigma^{\prime} / \sigma=0.45, k_{\mathrm{B}} T / \epsilon=0.1$, and $\epsilon^{\prime} / \epsilon=10^{3}$. To see the higher-order peak clearly, we show $2 \pi r \delta r g(r)$ in the vertical axis. The data are the averages of 10 samples.

and $\mathrm{b}$, is $r_{2}=\sqrt{3} r_{0}$, and that between the third nearest neighbors, e. g., the particles $\mathrm{a}$ and $\mathrm{c}$, and the particles a and $\mathrm{e}$, is $r_{3}=2 r_{0}$. The numbers of connections with the first, second, and third nearest neighbors in the minimum unit are $N_{1}=1, N_{2}=1$, and $N_{3}=3$, respectively. Taking into account the total particle number in the minimum unit, the interaction energy per particle $E^{\mathrm{R}}$ is estimated as

$$
\begin{aligned}
\frac{E^{\mathrm{R}}}{4 \epsilon} & =N_{1} \frac{\epsilon^{\prime}}{\epsilon}\left[\left(\frac{\sigma^{\prime}}{r_{0}}\right)^{12}-\left(\frac{\sigma^{\prime}}{r_{0}}\right)^{6}\right]+N_{2} \frac{\epsilon^{\prime}}{\epsilon}\left[\left(\frac{\sigma^{\prime}}{\sqrt{3} r_{0}}\right)^{12}-\left(\frac{\sigma^{\prime}}{\sqrt{3} r_{0}}\right)^{6}\right] \\
& +N_{3}\left[\left(\frac{\sigma}{2 r_{0}}\right)^{12}-\left(\frac{\sigma}{2 r_{0}}\right)^{6}\right] .
\end{aligned}
$$

Figure 8(b) shows the structure of the honeycomb lattice expected from Fig. 4(a). The first, second, and third nearest neighbors are, e.g., the particles a and b, the particles $\mathrm{a}$ and $\mathrm{c}$, and the particles a and $\mathrm{d}$, respectively. In the lattice, their distances are given by $r_{1}=r_{0}, r_{2}=\sqrt{3} r_{0}$, and $r_{3}=2 r_{0}$. The numbers of connections are given by $N_{1}=3$, 

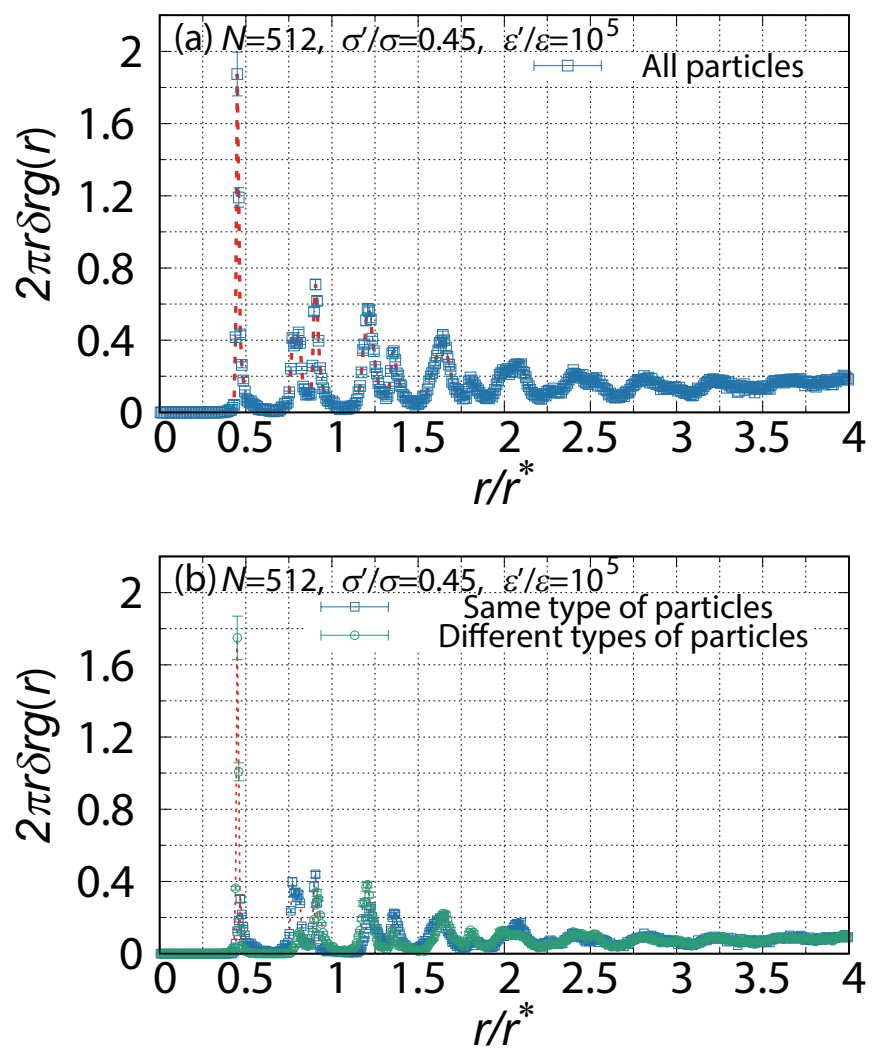

Fig. 7. (color online) (a) Radial distribution function $g(r)$ and (b) the contributions of the same and different species to $g(r)$, where $\sigma^{\prime} / \sigma=0.45, k_{\mathrm{B}} T / \epsilon=0.1$, and $\epsilon^{\prime} / \epsilon=10^{5}$. The data are the averages of 10 samples.

$N_{2}=6$, and $N_{3}=3$. Thus, the interaction energy per particle $E^{\mathrm{H}}$ is estimated as

$$
\begin{aligned}
\frac{E^{\mathrm{H}}}{4 \epsilon} & =\frac{1}{2}\left\{N_{1} \frac{\epsilon^{\prime}}{\epsilon}\left[\left(\frac{\sigma^{\prime}}{r_{0}}\right)^{12}-\left(\frac{\sigma^{\prime}}{r_{0}}\right)^{6}\right]+N_{2}\left[\left(\frac{\sigma}{\sqrt{3} r_{0}}\right)^{12}-\left(\frac{\sigma}{\sqrt{3} r_{0}}\right)^{6}\right]\right. \\
& \left.+N_{3} \frac{\epsilon^{\prime}}{\epsilon}\left[\left(\frac{\sigma^{\prime}}{2 r_{0}}\right)^{12}-\left(\frac{\sigma^{\prime}}{2 r_{0}}\right)^{6}\right]\right\} .
\end{aligned}
$$

Figure 8(c) shows the structure of the square lattice expected from Fig. 4(b). In the lattice, we do not need to take into account the effect of the third nearest neighbors. The first and second nearest neighbors are, e.g., the particles a and b, and the particles a and $\mathrm{c}$, respectively. Their distances are given by $r_{1}=r_{0}$ and $r_{2}=\sqrt{2} r_{0}$, and the numbers of connections are $N_{1}=2$ and $N_{2}=2$, so that the interaction energy per particle $E^{\mathrm{S}}$ is estimated as

$$
\frac{E^{\mathrm{S}}}{4 \epsilon}=N_{1} \frac{\epsilon^{\prime}}{\epsilon}\left[\left(\frac{\sigma^{\prime}}{r_{0}}\right)^{12}-\left(\frac{\sigma^{\prime}}{r_{0}}\right)^{6}\right]+N_{2}\left[\left(\frac{\sigma}{\sqrt{2} r_{0}}\right)^{12}-\left(\frac{\sigma}{\sqrt{2} r_{0}}\right)^{6}\right] .
$$




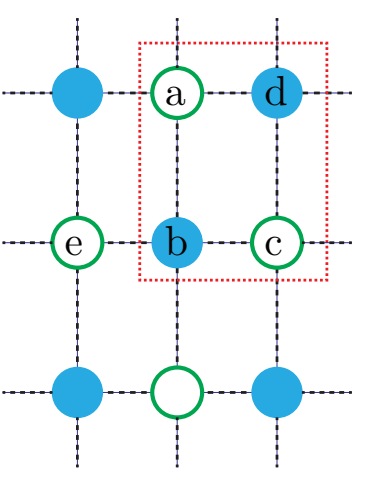

(a)

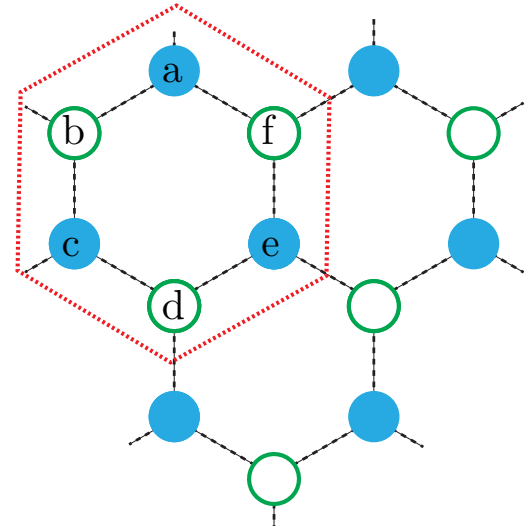

(b)

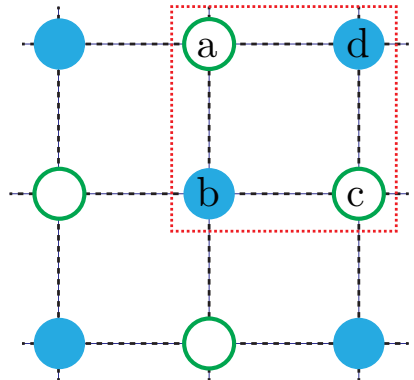

(c)

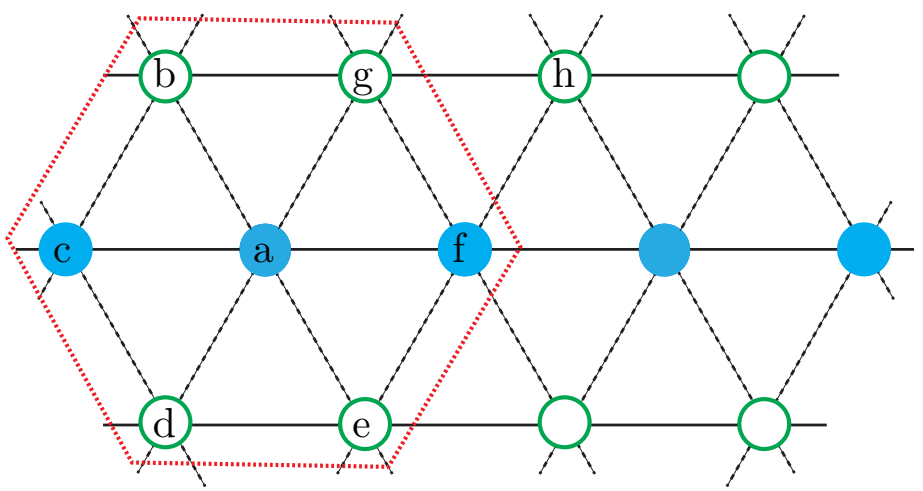

(d)

Fig. 8. (color online) Lattice structures formed by two species: (a) rectangular lattice, (b) honeycomb lattice, (c) square lattice, and (d) triangular lattice, where we distinguish the two species from their colors. The minimum unit is surrounded by red dotted lines. Black dashed lines represent the bonds between the different species.

Finally, we consider the lattice structure expected from Fig. 4(c). Figure 1 shows that $\phi_{6}$ is the largest parameter when $\epsilon^{\prime} / \epsilon=10^{5}$; thus, we expect the formation of the triangular lattice shown in Fig. 8(d) with a low energy because the contribution of the same species to the first peak is larger than that of the different species in Fig. 7. The first and second nearest neighbors are, e.g., particles a and b, and particles b and 
d, respectively. There are two types of third nearest neighbors. One includes the third nearest neighbors in the minimum unit such as the particles $c$ and $f$, and the other induces the third nearest neighbors across the minimum units such as the particles $b$ and $h$. In the lattice, $r_{1}=r_{0}, r_{2}=\sqrt{3} r_{0}$, and $r_{3}=2 r_{0}$. When we count the numbers of connections, we need to take into account the types of species The numbers of the connections with the first, second, and third nearest neighbors consisting of the same species are $N_{1}=3, N_{2}=3$, and $N_{3}=6$, respectively, and those consisting of different species are $N_{1}^{\prime}=6, N_{2}^{\prime}=6$, and $N_{3}^{\prime}=0$, respectively. Thus, the interaction energy per particle $E^{\mathrm{T}}$ is estimated as

$$
\begin{aligned}
\frac{E^{\mathrm{T}}}{4 \epsilon} & =\frac{1}{3}\left\{N_{1}\left[\left(\frac{\sigma}{r_{0}}\right)^{12}-\left(\frac{\sigma}{r_{0}}\right)^{6}\right]+N_{1}^{\prime} \frac{\epsilon^{\prime}}{\epsilon}\left[\left(\frac{\sigma^{\prime}}{r_{0}}\right)^{12}-\left(\frac{\sigma^{\prime}}{r_{0}}\right)^{6}\right]\right. \\
& +N_{2}\left[\left(\frac{\sigma}{\sqrt{3} r_{0}}\right)^{12}-\left(\frac{\sigma}{\sqrt{3} r_{0}}\right)^{6}\right]+N_{2}^{\prime} \frac{\epsilon^{\prime}}{\epsilon}\left[\left(\frac{\sigma^{\prime}}{\sqrt{3} r_{0}}\right)^{12}-\left(\frac{\sigma^{\prime}}{\sqrt{3} r_{0}}\right)^{6}\right] \\
& \left.+N_{3}\left[\left(\frac{\sigma}{2 r_{0}}\right)^{12}-\left(\frac{\sigma}{2 r_{0}}\right)^{6}\right]+N_{3}^{\prime} \frac{\epsilon^{\prime}}{\epsilon}\left[\left(\frac{\sigma^{\prime}}{2 r_{0}}\right)^{12}-\left(\frac{\sigma^{\prime}}{2 r_{0}}\right)^{6}\right]\right\}
\end{aligned}
$$

Figure 9 shows the energies estimated using Eqs. (8)-(10). The structure with the minimum interaction energy is the rectangular lattice when $\epsilon^{\prime} / \epsilon<70$, the honeycomb lattice when $70<\epsilon^{\prime} / \epsilon<800$, the square lattice when $800<\epsilon^{\prime} / \epsilon<10^{5}$, and the triangular lattice when $10^{5}<\epsilon^{\prime} / \epsilon$. The relationship between the lattice structures with the minimum interaction energy and the ratio $\epsilon^{\prime} / \epsilon$ roughly agrees with our simulations.

\section{Summary}

In this paper, keeping the two-dimensional lattice formed by DNA nanoparticle in mind, we studied the effect of the difference in interaction strength on the lattice structures. Assuming that $\epsilon^{\prime}$ is larger than $\epsilon$, we carried out Brownian dynamics simulations. With increasing $\epsilon^{\prime}$, the rectangular structure formed with $\sigma^{\prime} / \sigma=0.45$ changes to the honeycomb lattice, square lattice, and triangular lattice in this sequence. The change in the lattice structure induced by increasing $\epsilon^{\prime}$ is explained by the simple estimation of the interaction energy of each structure. We also carried out similar simulations in the cases that $\sigma^{\prime} / \sigma=0.58$ and 0.71 although we did not show the results. When $\sigma^{\prime} / \sigma=0.58$, the honeycomb structure, which is formed when $\epsilon^{\prime} / \epsilon=1$, changes to the square lattice and triangular lattice in succession with increasing $\epsilon^{\prime} / \epsilon$. The transition of the square lattice to the triangular lattice induced by the increase in $\epsilon^{\prime} / \epsilon$ also occurs in the case that $\sigma^{\prime} / \sigma=0.71$. For each $\sigma^{\prime} / \sigma$, the structure changes to the triangular lattice with a 

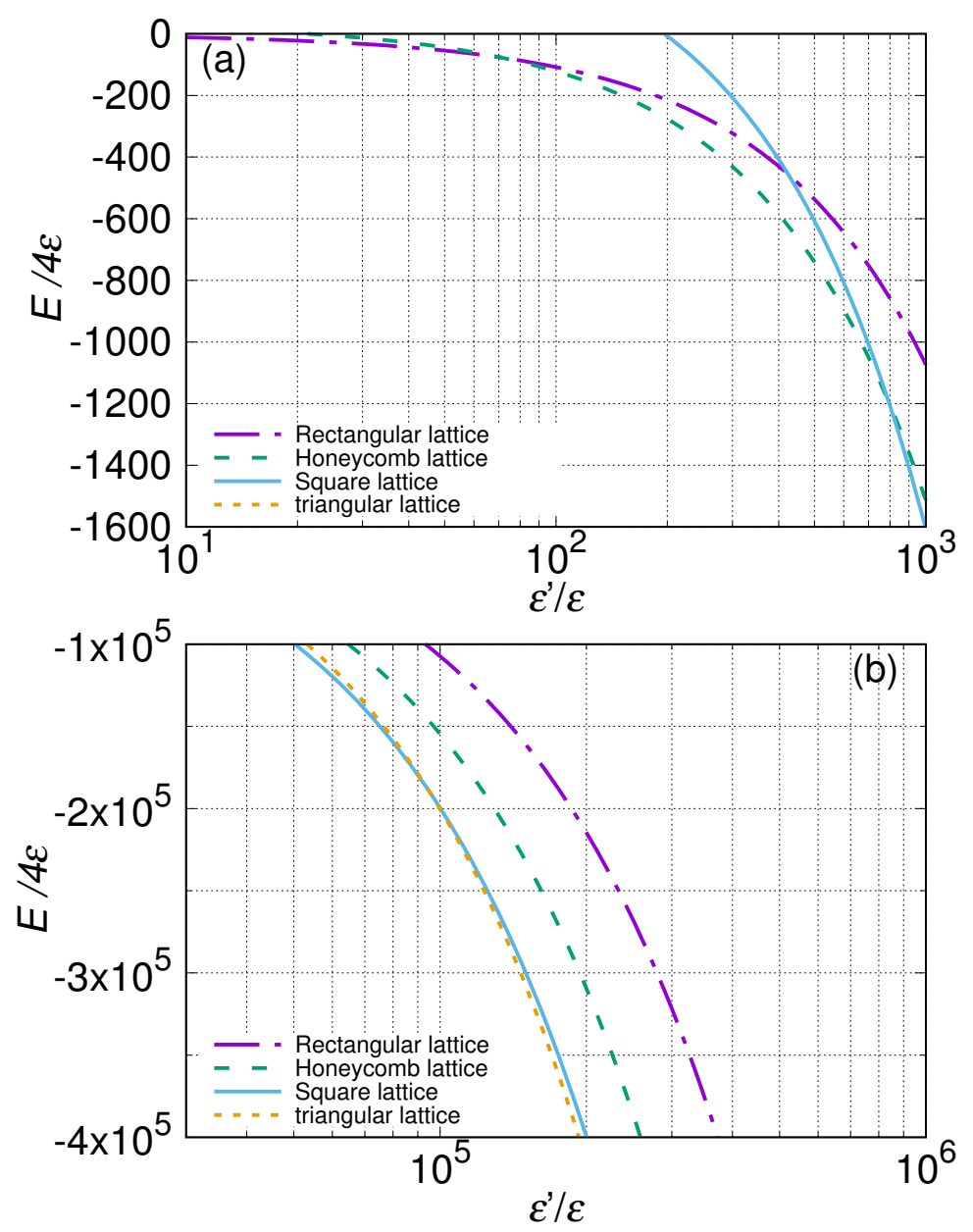

Fig. 9. (color online) Dependences of $E^{\mathrm{R}} / 4 \epsilon, E^{\mathrm{H}} / 4 \epsilon, E^{\mathrm{S}} / 4 \epsilon$, and $E^{\mathrm{T}} / 4 \epsilon$ on $\epsilon^{\prime} / \epsilon$. The $\epsilon^{\prime} / \epsilon$ ranges are (a) $10<\epsilon^{\prime} / \epsilon<10^{3}$ and (b) $10^{4}<\epsilon^{\prime} / \epsilon<10^{6}$.

sufficiently large $\epsilon^{\prime}$.

In our simulations, instead of decreasing $\epsilon$ and temperature tuning, we increased $\epsilon^{\prime}$ with the temperature fixed. In our previous study, ${ }^{8}$ we showed that not only the ratio of the interaction length $\sigma^{\prime} / \sigma$ but also the ratio of the interaction strength $\epsilon^{\prime} / \epsilon$ are important for determining the lattice structure. The effect of increasing $\epsilon^{\prime} / \epsilon$ is the same as that of increasing $\sigma^{\prime} / \sigma$. From our previous study, ${ }^{8}$ we found that increasing $\sigma^{\prime} / \sigma$ can change the square lattice to the triangular lattice. In this study, increasing $\epsilon^{\prime}$ changes the lattice structure from the honeycomb lattice to the triangular lattice via the square lattice when $\sigma^{\prime} / \sigma=0.45$. Increasing $\epsilon^{\prime} / \epsilon$ changes the lattice structures to the triangular lattice when $\sigma^{\prime} / \sigma \neq 1$. The triangular lattice is formed when $\sigma^{\prime} / \sigma=1$ and $\epsilon^{\prime} / \epsilon=1$, so that increasing $\epsilon^{\prime} / \epsilon$ does not cause the change in the two-dimensional structure when $\sigma^{\prime} / \sigma=1$. From our studies, it is not sufficient to determine the validity 
of Isogai's proposal that bundling the DNA strands causes the change in the twodimensional lattice structure. However, if the difference in interaction length is induced by controlling the magnesium ion density in a solution, it is possible to induce the change in the lattice structure without bundling the DNA strands. The lattice constant is changed in Ref. 7, so that the change in the lattice structure induced by $\sigma^{\prime} / \sigma$, which is shown in Ref. 8, agrees with the experiment qualitatively.

In colloidal dispersion systems, long-range attractions such as depletion attraction and Van der Waals interaction act between particles in general. Thus, we think that it is natural to take into account a long-range interaction in our simulation. ${ }^{8}$ However, it might be better to add the short-range interaction potential, which represents the attraction by DNA strands, to other long-range attractive potentials than using the modified LJ potential. Nevertheless, as a first step of the study, we used the same model as that in our previous study and investigated the effect of the strength of the interaction potential, which we neglected before. It is very important to study how the form of the interaction potential affects the lattice structure. Considering more suitable potential and studying its effect on the lattice structure are our future problems.

\section{Acknowledgements}

This work is supported by JSPS KAKENHI Grant Numbers JP26390054 and JP16K05470. We acknowledge the support from the Joint Research Program of the Institute of Low Temperature Science, Hokkaido University. 


\section{References}

1) D. Nykypanchuk, M. M. Maye, D. van der Lelie, and O. Gang, Nature 451, 549 (2008).

2) S. Y. Park, A. K. R. Lytton-Jean, B. Lee, S. Weigand, G. C. Schatz, and C. A. Mirkin, Nature 451, 553 (2008).

3) R. J. Macfarlane, B. Lee, H. D. Hill, A. J. Senesi, S. Seifert, and C. A. Mirkin, Proc. Natl. Acad. Sci., U.S.A. 106, 10493 (2009).

4) R. J. Macfarlane, B. Lee, M. R. Jones, N. Harris, G. C. Schatz, and C. A. Mirkin, Science 334, 204 (2011).

5) C. Zhang, R. J. Macfarlane, K. L. Young, C. H. J. Choi, L. Hao, E. Auyeung, G. Liu, X. Zhou, and C. A. Mirkin, Nature mater. 12, 741 (2013).

6) T. Isogai, A. Piednoir, E. Akada, Y. Akahosi, R. Tero, S. Harada, T. Ujihara, and M. Tagawa, J. Cryst. Growth 401, 494 (2014).

7) T. Isogai, E. Akada, S. Nakada, N. Yoshida, R. Tero, S. Harada, T. Ujihara, and M. Tagawa, Jpn. J. Appl. Phys. 55, 03DF11 (2016).

8) H. Katsuno, Y. Maegawa, and M. Sato, J. Phys. Soc. Jpn. 85, 074605 (2016).

9) D. L. Ermak, J. Chem. Phys. 62, 4189 (1975).

10) B. I. Halperin and David R. Nelson, Phys. Rev. Lett. 41, 121 (1978).

11) R. Yamamoto and A. Onuki, J. Phys. Soc. Jpn. 66, 2545 (1997).

12) R. Yamamoto and A. Onuki, Phys. Rev. E 58, 3515 (1998).

13) T. Hamanaka and A. Onuki, Phys. Rev. E 74, 011506 (2006).

14) M. Sato, H. Katsuno, and Y. Suzuki, Phys. Rev. E 87, 032403 (2013). 